\title{
A method for the accurate quantification of gas streams by on-line mass spectrometry
}

\author{
Edwing Alexander Velasco-Rozo ${ }^{1}$, Luz Marina Ballesteros-Rueda ${ }^{1}$, Víctor Gabriel Baldovino- \\ Medrano ${ }^{1,2, *}$ (iD. \\ ${ }^{1}$ Centro de Investigaciones en Catálisis (CICAT), Escuela de Ingeniería Química, Universidad Industrial de Santander, Parque Tecnológico \\ de Guatiguará, Km 2 vía El Refugio, Piedecuesta, Santander 681011, Colombia. \\ ${ }^{2}$ Laboratorio de Ciencia de Superficies (SurfLab), Universidad Industrial de Santander, Parque Tecnológico de Guatiguará, Km 2 vía El \\ Refugio, Piedecuesta, Santander 681011, Colombia. \\ *Corresponding author. Tel. +54 6344000 ext. 1485. Email: vicbaldo@ uis.edu.co. ORCID: http://orcid.org/0000-0003-3227-0251. Twitter: \\ @ vigabalme
}

\begin{abstract}
The accuracy of the online quantification of gaseous effluents from catalytic reactors by mass spectrometry (MS) is rarely addressed by researchers, despite the extensive use of the technique. MS results are strongly sensitive to both the operation conditions of the reactor and to the state of the instrument. Therefore, most studies use them as qualitative descriptors of the performance of catalytic reaction systems. The purpose of this work was to develop an accurate method for the quantification of gaseous effluents from catalytic reactors. For this purpose, the mathematical expressions from the so-called external and internal standard calibration methods for MS were coupled to the typical metrics used for studying catalytic reactions; namely, conversion, selectivity, and carbon mass balances. The catalytic combustion of methane was selected as a model reaction to test the developed approach. The accuracy of the developed method was validated by comparison with results obtained in a separated reaction system coupled online to a gas chromatograph. The closure of the carbon mass balance was used as a control metrics allowing for the assessment of the physical meaning of the method. In general, the internal standard method of calibration was found to be best for the accurate quantification of gaseous streams by on-line mass spectrometry. In general, the results of this investigation may be of use to researchers in the field of catalysis as well as to research workers using mass spectrometry for various purposes.
\end{abstract}

Keywords: Accurate quantification; On-line MS analysis; Internal-External standard methods; Gas phase catalytic reactions; Carbon mass balance

\section{Introduction}

Further fundamental and technological developments on catalytic gaseous phase reactions such as hydrocarbon reforming and partial or total oxidation of organic volatile compounds are environmentally strategical to cope with climate change and for controlling the emission of hazard compounds.[1,2] Basic research on this field aims at understanding the effects of the reaction conditions; temperature, composition, and space velocity, over the catalytic 
performance and over the designs of both the reactor and the catalysts themselves. The most common metrics for assessing the catalytic performance are the conversion of reactants and the yields and selectivities to the reaction products[3-5]. These metrics are normally the base for assessing the thermodynamics, transport phenomena, and kinetics of the system and their further correlation with the physicochemical properties of the catalyst and with their evolution with time on stream. The calculation of the former is rather straightforward given that the composition of the gaseous effluents from the reactor is determined. Therefore, the accuracy of the instrumental set-up implemented for their quantification defines the accuracy of the metrics used to evaluate the catalytic performance.

On-line techniques such as gas chromatography (GC) and mass spectrometry (MS) are common for quantifying gaseous streams [6,7]. In general, gas chromatography is highly versatile but conventional instruments require methods whose analysis time may range between minutes and a short couple of hours. Furthermore, multiple detectors arranged in special configurations may be required for the analysis of all the components in a multicomponent effluent. On the other hand, on-line mass spectrometry can potentially overcome the above problems thanks to its capacity to provide structural information and product composition of multicomponent systems in analysis times on the order of seconds to minutes.

A given analyte studied by conventional mass spectrometers undergoes three basic processes inside the instrument. First, the analyte if submitted to fragmentation/ionization by the impact of high energy electrons. Second, the produced ions are fileted according to the scanning of a predefined a mass to charge, $\mathrm{m} / \mathrm{z}$, range. And, third, the selected ions are detected.[8] In general, the software developed for data acquisition and analysis plays a key in the detection and identification of the compounds from the gaseous stream being analyzed. For quantification, Olivares [9] that the analysis can be based on the proportionality between the partial pressures of the components from the analyzed mixture and the intensity of the signals for the ions read in the MS spectrum. However, phenomena such as overlapping of signals coming from two or more compounds totally or partially sharing fragmentation patterns, and the dependence of signals intensities on the temperature and pressure of the monitored system and on the evolution of the internal parts of the mass spectrometer; e.g. the filament, may make quantification so difficult that often researchers opt for interpreting MS data only from a qualitative standpoint.

To overcome the issues of MS quantification, some authors [9-11] have proposed methods that allow to calculate the individual contribution of each compound to the MS spectrum. The methods comprise the solution of an algebraic interval matrix formed by all the analytes of the effluent and their detected ions by assuming that there is a proportionality between the concentration of the former and the non-overlapping spectra of the latter. The proportionality factors; also named as sensitivity or calibration factors, can be calculated using defined calibration routines. There are two known calibration methods: namely, the external and the internal standard methods. $[12,13]$ The external standard method estimates the sensitivity factors by correlating MS signals to known gas concentrations via least-squares regressions. The internal standard method not only does the latter but also uses the 
signal from a given inert compound whose concentration remain constant and know during the performed tests to normalize the intensities of the other analytes. For on-line MS systems aimed at monitoring catalytic systems or related experimental set-ups, the internal standard method is recommended.

The aim of this contribution was to develop a reliable method for the highly accurate quantification of the composition of gaseous reaction effluents by online mass spectrometry. A particular challenge for this kind of applications is that both the concentration and nature of the components of effluent change over time due to the catalytic reaction. To tackle the effect that these changes have on the MS spectra, a mathematical model integrating the variables inherent to the evolution of the catalytic reaction and those comprised in the instrumental analysis was developed. Particularly, the analysis of mass balances was implemented as a control tool for checking both the accuracy and physical meaning of the developed method. This strategy has proved successful for improving the accuracy of on-line GC analysis [14] but, as far as the authors of this paper know, it had not been used as part of a method for accurately quantifying gaseous streams from catalytic systems by mass spectrometry. Herein, the catalytic combustion of methane over a continuous plug-flow bed reactor was used as a model reaction to test the accuracy of the mathematical methods developed from both the internal and the external standard methods of calibration. In general, the internal standard method produced the most accurate and physically meaningful MS quantification for the studied gaseous streams. To validate the results of the method, catalytic tests were also made in a different reaction set-up operated under identical reaction conditions but whose effluent was monitored by online gas chromatography.

\section{Theoretical background}

The response of a mass spectrometer in front of a gaseous stream is a distribution of current intensities for each one of the ions formed after the partition of each one of the components of the stream. The registered signals are showed in a software interface in the form of a distribution of $\mathrm{m} / \mathrm{z}$ ratios. When the instrument is coupled to the outlet of a continuous reactor, i.e. online analysis, its response is a function of the time on stream (TOS) of the catalytic system.[15] This implies that for each molecule or atom composing the outlet stream of the catalytic system and entering the spectrometer a variety of $\mathrm{m} / \mathrm{z}$ signals are found. These signals may overlap, but state of the art software [16-18] has allowed accurate compound identification. Furthermore, there are highly efficient instrumentation and software allowing completely automatized reactors coupled to online mass spectrometers. However, high precision quantification from $\mathrm{m} / \mathrm{z}$ signals and thus a satisfactory correlation with the molar flow of each component in the reactor stream remains poorly developed. In what follows, we discuss some aspects related to the challenges that are generally present in this kind of coupled online systems.

\subsection{Online mass spectrometry}


Online mass spectrometers are generally provided with a capillary connected directly to the outlet stream of the reactor set-up, as outlined in Figure 1. The internal diameter of the capillary is in the scale of microns and its length should be long enough to reduce the amount of matter entering the instrument while keeping its vacuum level. This item is the bridge between the reactor and the detection system. Normally, at the end of the capillary, the analyte, herein a sample of the gaseous stream from the reactor outlet, passes to the low-pressure ion source via a ferrulesealed vacuum feedthrough. Once the sample is inside the mass spectrometer, it is ionized and fragmented by the bombardment of high energy electrons emitted by a hot wire filament. The latter is the most common and maybe the oldest method for ionization, but it is not unique [19,20]. After that, the ions are directed to the quadrupole mass analyzer. This consists of four parallel rods placed at the corner of a square. The principal function of the mass analyzer is to filter and to accelerate the ions. This is done by quickly modifying the direct current and radiofrequency voltages in a way in which only a selected mass to charge ratio $(\mathrm{m} / \mathrm{z})$ goes through the quadrupole while other ions collide with the rods or are accelerated out of the array. The process is repeated for a wide range of $m / z$ in a very short time thus allowing the ions passing to the detector once at a time.

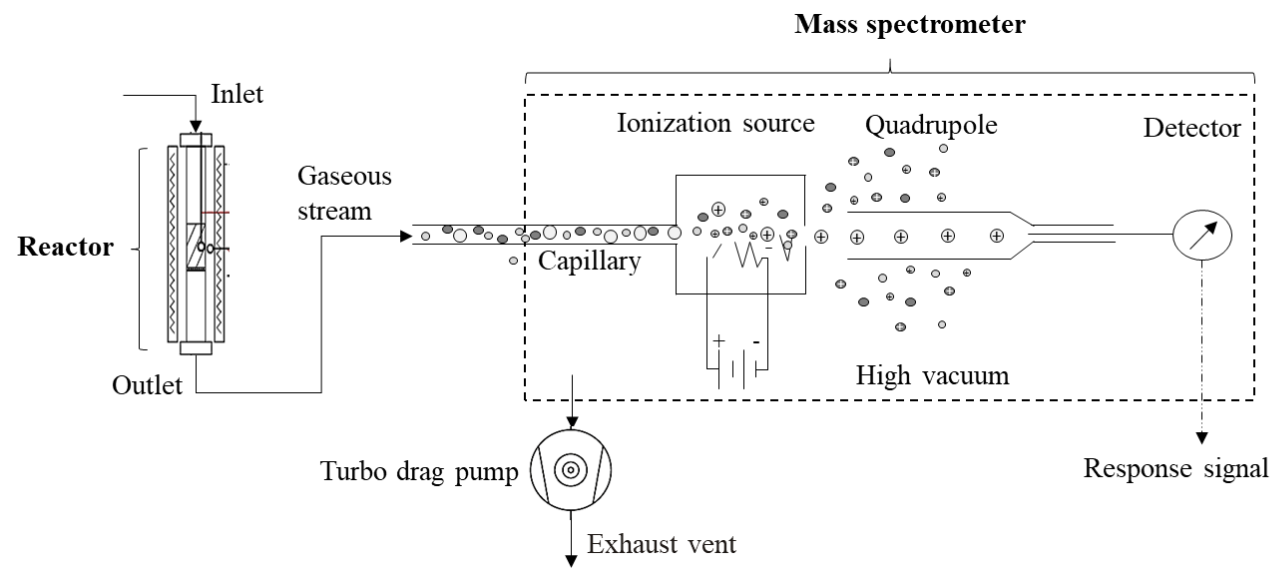

Figure 1. Scheme for an online mass spectrometer.

Typically, there are two types of detectors at the end of the instrument: the Faraday cup and the Secondary Electron Multiplier (SEM). The Faraday cup works with high accuracy in concentrations above the parts per millions of gas at atmospheric pressure. Low cost, simplicity, accuracy, and low noise are among its most representative characteristics. Its functioning is simple; when the accelerated ions beam collides with a metallic electrode it generates a current that after passing through a grounded high impedance resistor then generates a voltage that is read as the signal by the instrument. On the other hand, SEM detectors are sensitive to concentrations of parts per billion. The larger sensitivity of these detectors is due to the use of dynodes that successively amplify secondary electrons generated by the collision of the ions beam with an electron emissive surface. The multiplied electrons cascade is finally guided to a receptor electrode where a response signal is generated. In systems provided with coupled Faraday cup and SEM detectors, generally the SEM is used when the Faraday detection sensibility is not 
enough. Overall, detectors generate an ion current response $\left(I_{i j}\right)$ for the $\mathrm{i}^{\text {th }} \mathrm{m} / \mathrm{z}$ of the $\mathrm{j}^{\text {th }}$ gaseous compound analyzed, Equation 1:[20,21]

$$
I_{i j}=G_{i} I_{e} \sigma_{i} n_{i}
$$

Where, $G_{i}$ is the transmission efficiency for a given ion, $I_{e}$ is the electron beam current, $\sigma_{i}$ is the cross-section for ionization, and $n_{j}$ is the number density of target molecules (or atoms). Equation. 1 relates the current detected for a specific ion with the number of molecules (or atoms) that generated these ions in the ionization source. At conditions of ionization, $n_{i}$ can be related to the total pressure in the ionization source $\left(P_{T, I S}\right)$, Equation 2.

$$
n_{i}=P_{j, I S} / T_{I S} a
$$

Where, $a$ is a constant and $T_{I S}$ is the effective temperature in the ionization region. Equations 1 and 2 can be rewritten to relate linearly the ion current response with the partial pressure, Equation 3. Notice that this expression assumed a constant transmission efficiency, an electron beam current for a specified $\mathrm{m} / \mathrm{z}$, and a constant temperature in the ionization region.

$$
I_{i j}=L_{i j} P_{j, I S}
$$

Where, $L_{i j}$, is an integrated calibration factor commonly named as instrument sensitivity. In a multicomponent gaseous mixture, the measured ion current $\left(I_{i}\right)$ of each $\mathrm{i}^{\text {th }} \mathrm{m} / z$ can be described as the sum of the individual contributions of each $\mathrm{j}^{\mathrm{th}}$ gaseous compound fragmented at this mass to charge, Equation 4:

$$
I_{i}=\sum_{j} I_{i j}=\sum_{j} L_{i j} P_{j, I S}
$$

According to Ellefson et al. [21], $P_{j, I S}$ is proportional to the pressure of $\mathrm{j}^{\text {th }}$ gaseous compound in the gaseous stream source $\left(P_{j}\right)$, such as $P_{j}$ is equivalent to the pressure on the outlet of the reactor in online systems, see Figure 1. Hence, Equation 4 can be rewritten as Equation 5. By assuming the above and an ideal gas behavior, Equation 4 can also be rewritten in terms of the molar flow of a $\mathbf{j}^{\text {th }}$ gaseous compound $\left(F_{j}\right)$ :

$$
I_{i}=\sum_{j} I_{i j}=\sum_{j} L_{i j} P_{j} \quad \text { or } \quad I_{i}=\sum_{j} I_{i j}=\sum_{j} l_{i j} F_{j}
$$

Where, $l_{i j}$ is the sensitivity factor affected by the proportionality between the pressure and the molar flow. On the other hand, since usually the initial mass spectrometer responses are always different to zero, and, since Equation 1 does not include an initial value, it is necessary to apply a correction to Equation 5, by including the real mass spectrometer response, $I_{i, j}^{\text {real }}$, and its background response, $I_{i, j}^{B G}$, Equation 6.

$$
I_{i, j}=I_{i, j}^{r e a l}-I_{i, j}^{B G}
$$


It can be said that the previous analysis is just a mathematical development to achieve the adaptation of the BeerLambert-Bouguer law [22] to an online mass spectrometer. The literature[23,24] presents similar procedures that relate the molar flow at the outlet of the reactor with the mass spectrometer signal.

\subsubsection{Online mass spectrometer calibration}

Calibration routines are carried out to estimate sensitivity factors relating the mass spectrometer response signal for a given $\mathrm{j}$ component with its molar flow (pressure or concentration). According to Equation 5 , there are $i x j$ sensitivity factors; that is, one for each fragment of each gas in the multicomponent mixture. To avoid the excessive experimentation and cost of implementing a calibration routine for determining all sensitivity factors, it is possible to use the following expression to calculate the minor fragments starting from the main fragment, i.e. the highest $\mathrm{m} / \mathrm{z}$ detected for each gas, Equation 7:

$$
l_{i j}=I_{R, i j} l_{i j}^{\text {main }}
$$

Where, $l_{i j}^{\text {main }}$ is the sensitivity factor for the main fragment, and $I_{R, i j}$ is the relative intensity of the $\mathrm{i}^{\text {th }}$ fragment from the main fragment for compound $j$ which can be gotten from the database of mass spectrometry. Generally, the mass spectrometer software includes a database for these relative intensities. There are two known ways to calculate the sensitivity factor for the main fragment: namely, the external standard and internal standard methods.

External standard method. In this method, the sensitivity factor for the main fragment is calculated by the determination of the slope of a linear regression of the molar flow (partial pressure or concentration) from the gas stream as a function of the intensity of the signal of the main fragment of each gas in the multicomponent mixture.[25] For this purpose, gaseous mixtures of different and known compositions are analyzed by the mass spectrometer. After calibration, the sensitivity factors of the minor fragments are calculated with Equation 7 and this equation is replaced in Equation 5. With the latter, an algebraic expression to calculate the molar flow from a detected response signal of a gas compound is obtained.

Internal standard method. This method normalizes the mass spectrometer response signal for a compound $\mathrm{j}$ in terms of the response signal from the main fragment of a given standard compound $\left(I_{s t d}\right)$. Of course, the standard must remain inert under the studied experimental conditions.[12] Normalization allows compensating the effects generated by the chemical transformation occurring in the catalytic reactor on the flow entering the mass spectrometer[26]. Therefore, the sensitivity factor for the main fragment is calculated with the slope of the linear regression of the normalized data of the molar flow (partial pressure or concentration), $\left(\frac{I_{i, j}}{I_{s t d}}\right)$ as a function of the response signal of the main fragment $\left(\frac{F_{j}}{F_{s t d}}\right)$ for each gas in the mixture. After calibration, sensitivity factors of 
minor fragments are calculated by Equation 7 and replaced in Equation 8 to obtain an algebraic expression to calculate the molar flow from a detected response signal of a gas compound.

$$
\frac{I_{i, j}}{I_{s t d}}=l_{i j} \frac{F_{j}}{F_{s t d}}
$$

\subsection{Characterization of the behavior of a catalytic reactor with online mass spectrometry}

The performance of a catalytic reactor is rather straightforwardly assessed by two metrics: namely, conversion $\left(X_{r}\right)$ and overall selectivity $\left(S_{p}\right) \cdot[3,4,27]$. Conversion refers to the change in the amount of a determined reactant due to its transformation during a chemical reaction. It may be calculated as the difference between the molar flow at the inlet and at the outlet of the reactor, Equation 9:

$$
X_{r}=\frac{F_{r}^{0}-F_{r}}{F_{r}^{0}}
$$

On the other hand, the overall selectivity expresses the relative amount of a given product as referred to the other products, Equation 10:

$$
S_{p}=v \frac{F_{p}-F_{p}^{0}}{F_{r}^{0}-F_{r}}
$$

In Equations 9 and 10, $F_{r}^{0}$ and $F_{r}$ are the molar flows of the reactants at the inlet and at the outlet of the reactor, respectively, $F_{p}^{0}$ and $F_{p}$ are the molar flows of products at the inlet and at the outlet of the reactor, respectively, and $v$ is the ratio between the stoichiometric coefficients of the reactants and products. Conveniently, conversion and selectivity can be rewritten in terms of the mass spectrometer response signal. For the reactor effluents, considering that there is no overlap between the fragments generated for each gas, the external and internal standard methods produce the following relationships:

$$
\begin{array}{llrl}
\text { External standard } & I_{r}=l_{r} F_{r} & \text { or } \quad I_{p}=l_{p} F_{p} \\
\text { Internal standard } & \frac{I_{r}}{I_{s t d}}=l_{r} \frac{F_{r}}{F_{s t d}} \quad \text { or } \quad \frac{I_{p}}{I_{s t d}}=l_{p} \frac{F_{p}}{F_{s t d}}
\end{array}
$$

Where, the nomenclature for the gaseous compounds is now referred to reactants, $r$, and products, $p$. Replacing equations 11 and 12 in equations 9 and 10 leads to the following:

$$
\begin{aligned}
& \text { External standard } \\
& \qquad \begin{aligned}
X_{r} & =\frac{I_{r}^{0}-I_{r}}{I_{r}^{0}} \\
S_{p} & =v \frac{l_{p}}{l_{r}} \frac{\left(I_{p}-I_{p}^{0}\right)}{\left(I_{r}^{0}-I_{r}\right)}
\end{aligned}
\end{aligned}
$$


Internal standard

$$
\begin{gathered}
X_{r}=\frac{I_{r}^{0} / I_{s_{s t d}^{0}}-I_{r} / I_{s t d}}{I_{r}^{0} / I_{s t d}^{0}} \\
S_{p}=v \frac{l_{p}}{l_{r}} \frac{\left({ }_{p}^{I_{p}} / I_{s t d}-{ }^{I_{p}^{0}} / I_{s t d}^{0}\right)}{\left(I_{r}^{0} / I_{s t d}^{0}-{ }^{I_{r}} / I_{s t d}\right)}
\end{gathered}
$$

Where, $I_{r}^{0}$ and $I_{p}^{0}$ are the mass spectrometer response signals for the reactants and products, respectively. For these expressions, $I_{r}^{0}$ and $I_{p}^{0}$ are referred to samples takes downstream of the reaction set-up, Figure 1.

On the other hand, when there is overlap between the fragments generated for each gas, it is necessary to solve the equation systems stemming from the Matrixes 1 or 2, according to the selected calibration method, to calculate the molar flows of reactants and products:

External standard

$$
\left|\begin{array}{cccc}
l_{i j} & l_{i j+1} & \cdots & l_{i n} \\
l_{i+1 j} & l_{i+1 j+1} & \cdots & l_{i+1 n} \\
\cdots & \cdots & \cdots & \cdots \\
l_{m j} & l_{m j+1} & \cdots & l_{m n}
\end{array}\right| \times\left|\begin{array}{c}
F_{j} \\
F_{j+1} \\
\cdots \\
F_{n}
\end{array}\right|=\left|\begin{array}{c}
I_{i} \\
I_{i+1} \\
\cdots \\
I_{m}
\end{array}\right|
$$

Internal standard

$$
\left|\begin{array}{cccc}
l_{i j} & l_{i j+1} & \cdots & l_{i n} \\
l_{i+1 j} & l_{i+1 j+1} & \cdots & l_{i+1 n} \\
\cdots & \cdots & \cdots & \cdots \\
l_{m j} & l_{m j+1} & \cdots & l_{m n}
\end{array}\right| \times\left|\begin{array}{c}
\frac{F_{j}}{F_{s t d}} \\
\frac{F_{j+1}}{F_{s t d}} \\
\cdots \\
\frac{F_{n}}{F_{s t d}}
\end{array}\right|=\left|\begin{array}{c}
\frac{I_{i}}{I_{s t d}} \\
\frac{I_{i+1}}{I_{s t d}} \\
\cdots \\
\frac{I_{m}}{I_{s t d}}
\end{array}\right|
$$

(Matrix 2)

Where, $m$ and $n$ are the total $\mathrm{m} / \mathrm{z}$ fragments and the total gaseous compounds in the multicomponent mixture, respectively. To avoid including terms for the component used as the internal standard in matrix 2, the fragments from the internal standard must not overlap the fragments from any other compound.

\subsection{Application of mass balances to improve the accuracy of the quantification of gaseous streams with online mass spectrometry.}

In principle, online mass spectrometry is ideal for monitoring catalytic reactions because all the compounds that inlet and outlet the reactor can be detected. This allows implementing mass balances as a tool for improving the accuracy of the quantification made by the technique. A mass balance is based on the principle of mass conservation (that is always true if nuclear reactions are not involved). Therefore, the mass of the reactants entering the reactor equals the mass of the products at its outlet. Herein, this principle is applied by means of a metric called the mass balance coefficient $(\theta)$ that relates the masses of the reactants to the masses of the products, Equation 17:[14] 


$$
\frac{\sum\left[F_{r}-F_{r}^{0}\right] \overline{P M}_{r}}{\sum\left[F_{p}-F_{p}^{0}\right] \overline{P M}_{p}}=\theta
$$

Where, $\overline{P M}_{r}$ and $\overline{P M}_{p}$ are the molar weights of the reactants and products, respectively. Of course, the maximum value of $\theta$ is 1 for this value corresponds to a full closure of the mass balance. In this sense, when $\theta$ diverts from 1 a decrease in the accuracy of the quantification method is suggested when all other factors are considered equal. Equation 17 can be conveniently expressed in terms of the factors related to the mass spectrometer response signal by making adequate substitutions of Equations 11, or 12. One may notice that $\theta$ is a function of time and hence can provide information about the reliability and accuracy of the quantification process at all times and under all conditions of flow and temperature of the reaction test. On the other hand, Equation 17 could also be applied to each element that participate in the reaction; carbon balance, oxygen balance, among others. Further details on how to apply mass balances to improve the accuracy of online quantification systems is presented elsewhere. [14]

\section{Experimental}

The methods described above were used for the online quantification of the products from the catalytic combustion of methane, Equations 18 and 19. Catalytic tests were carried out using an automated microreactor system, CATLAB - PCS module 1 type 303230 (Hiden Analytical). The microreactor was coupled to a QGA mass spectrometer system type 305110 (Hiden Analytical). For comparison purposes, a set of reaction tests were done in another reaction set-up coupled online to a gas chromatograph. The specific details and the methods for quantification in the latter system are presented elsewhere. [14]

$$
\begin{aligned}
& \mathrm{CH}_{4}+2 \mathrm{O}_{2} \rightarrow \mathrm{CO}_{2}+2 \mathrm{H}_{2} \mathrm{O} \\
& \mathrm{CH}_{4}+\frac{3}{2} \mathrm{O}_{2} \rightarrow \mathrm{CO}+2 \mathrm{H}_{2} \mathrm{O}
\end{aligned}
$$

\subsection{Materials}

Methane $\left(\mathrm{CH}_{4}\right.$, Linde, purity of $\left.99.99 \%\right)$, oxygen $\left(\mathrm{O}_{2}\right.$, Linde, purity of $\left.99.994 \%\right)$, argon (Ar, Linde, Purity 99.998\%); used as a diluent, and helium (He, Linde, purity of 99.99\%); used as an internal standard, were used for the catalytic tests. For some experiments, nitrogen $\left(\mathrm{N}_{2}\right.$, Linde, Purity $\left.99.998 \%\right)$ was used as both diluent and internal standard. Commercial $\mathrm{MnO}_{2}$ (Erachem Comilog, LOT 622) was used as catalyst. The catalyst was diluted with inert quartz particles (Merck). Both the catalyst and the quartz particles were sifted to obtain particles of $\sim 75 \mu \mathrm{m}$. Glass wool, used to support the catalyst in the reactor, was provided by Merck.

\subsection{Description of the CATLAB set-up}

The microreactor module of the CATLAB set-up is provided with a quartz fixed-bed tubular microreactor of internal diameter equal to $0.6 \mathrm{~cm}$ placed inside of a tubular low thermal mass furnace controlled by a Eurotherm controller 
connected to a -in bed- sentry K-type thermocouple (with Cold Junction Compensation, Accuracy $+/-1{ }^{\circ} \mathrm{C}$, and Response $0.05 \mathrm{~s}$ ). The gases fed to the reactor are controlled by independent thermal mass flow controllers. At the outlet of the reactor, a sample of the reaction products is drawn down to a $2 \mathrm{~m}$ heated inert quartz capillary with heater supply operating up to $200{ }^{\circ} \mathrm{C}$ by the action of a sample bypass pumping line connected to a 60 liter per second turbomolecular pump. The capillary connects the reactor with an ultra-high vacuum (4.7 x $\left.10^{-7} \mathrm{mbar}\right)$ QGA mass spectrometer for the online analysis. The mass spectrometer is provided with an ion source, a quadrupole filter with a 300 amu mass range capability, and a dual Faraday/SEM detector having a detection limit around 100 ppb. Table 1 presents the parameters set for the QGA spectrometer in this work.

Table 1. Parameters set for the QGA spectrometer.

\begin{tabular}{lccccccc}
\hline Gas & Argon & Methane & Oxygen & Carbon dioxide & Carbon monoxide & Water & Helium \\
\hline Detector & Faraday & Faraday & Faraday & SEM & SEM & SEM & Faraday \\
Electron Energy & 70 & 70 & 70 & 70 & 70 & 70 & 70 \\
Emission Current & 400 & 350 & 400 & 300 & 400 & 400 & 250 \\
Start Range & $1 \times 10^{-7}$ & $1 \times 10^{-9}$ & $1 \times 10^{-7}$ & $1 \times 10^{-9}$ & $1 \times 10^{-9}$ & $1 \times 10^{-9}$ & $1 \times 10^{-9}$ \\
Auto Range Low & $1 \times 10^{-8}$ & $1 \times 10^{-12}$ & $1 \times 10^{-8}$ & $1 \times 10^{-12}$ & $1 \times 10^{-12}$ & $1 \times 10^{-12}$ & $1 \times 10^{-12}$ \\
Auto Range High & $1 \times 10^{-5}$ & $1 \times 10^{-7}$ & $1 \times 10^{-5}$ & $1 \times 10^{-7}$ & $1 \times 10^{-7}$ & $1 \times 10^{-7}$ & $1 \times 10^{-7}$ \\
\hline
\end{tabular}

For the catalytic tests, the catalytic bed was composed of a 1:10 weight ratio mixture of catalyst and quartz particles. The use of quartz as a diluent avoids artifacts due to temperature gradients. Further homogenization of the temperature of the reactor as well as reaching plug-flow in the system was achieved by placing a glass wool plug on top of the catalytic bed. The catalyst was dried in situ at $120{ }^{\circ} \mathrm{C}\left(5^{\circ} \mathrm{C} \mathrm{min}-1\right)$ for $90 \mathrm{~min}$ with a flow of argon at a space velocity of $78000 \mathrm{ml} \mathrm{g}^{-1} \mathrm{~h}^{-1}$. Then, still under argon flow, the reactor was allowed to cool down to $50{ }^{\circ} \mathrm{C}$ and then fed with a mixture of $\mathrm{CH}_{4}\left(7.7 \%\right.$ vol.), $\mathrm{O}_{2}(15.4 \%$ vol.), $\mathrm{He}(6 \%$ vol.), and argon as gas balance, keeping the same space velocity mentioned above. The temperature of the reactor was raised to $500{ }^{\circ} \mathrm{C}\left(5^{\circ} \mathrm{C} \min ^{-1}\right)$ either after $30 \mathrm{~min}$ or until complete stabilization of the flow fed to reactor. In a typical test, reaction products started to appear from $300{ }^{\circ} \mathrm{C}$. The reaction tests at $500^{\circ} \mathrm{C}$ were typically done for $6 \mathrm{~h}$ after which the reactor was again cooled to $50^{\circ} \mathrm{C}$ while keeping the flow of reactants. Finally, the reactor was kept at $50^{\circ} \mathrm{C}$ for $30 \mathrm{~min}$ to ensure the stability of the gas flowing to the mass spectrometer.

The analysis conditions for online mass spectrometry are shown in Table 1. Data acquisition was made by the CATLAB software. The following $\mathrm{m} / \mathrm{z}$ signals were followed: 44, 40, 32, 28, 18, 4 and 15, which were associated to the main fragments of $\mathrm{Ar}, \mathrm{CO}_{2}, \mathrm{O}_{2}, \mathrm{CO}, \mathrm{H}_{2} \mathrm{O}, \mathrm{He}$, and a minor fragment of $\mathrm{CH}_{4}$, respectively. The latter $\mathrm{m} / \mathrm{z}$ was chosen over the main $\mathrm{m} / \mathrm{z}$ line for $\mathrm{CH}_{4}$ at 16 to avoid overlap with the other lines such as $\mathrm{O}^{-}$. Before the catalytic test, calibration runs were performed at $50{ }^{\circ} \mathrm{C}$. These runs consisted of the analysis of gaseous mixtures with known compositions fed through the charged reactor in order to calculate sensitivity factors. Both the external and the 
internal standard calibration methods were applied using Equations 11 and 12. The recorded response signals and the calculated sensitivity factors were used to calculate reaction conversions and selectivities using Equations 1316. Mass balance coefficients were calculated for the carbon mass balance using Equation 17.

\subsection{Description of the OXIDATEST-online GC system set up}

Catalytic tests were also done in an automated reaction system, OXIDATEST, for validating the MS quantification method proposed herein. The OXIDATEST consists of a continuous flow stainless steel fixed bed reactor with an inner diameter of $10.4 \mathrm{~mm}$. The feed of the reactor was controlled by a set of independent mass flow controllers (Alicat Scientific) with an accuracy of $\pm 0.1 \%$ of full scale. The reactor temperature was controlled by a programmable logic controller (PLC, Rockwell Automation) coupled to a concentric tubular furnace and a thermocouple placed inside the catalytic bed. The outlet pressure of the reaction system was set at $110.3 \mathrm{kPa}$ using a back-pressure controller (PC, Alicat Scientific) with an accuracy of $\pm 0.3 \%$ of full scale. The outlet of the reactor was connected online to a Gas Chromatograph (GC-2014 instrument, Shimadzu Corporation) to make the online analysis of the reaction products. The instrument was provided with a set of packed columns and with a TCDMethanizer-FID array for detection and quantification. Samples from the gaseous stream from the reactor outlet, were analyzed every $20 \mathrm{~min}$. The catalytic reactor of the OXIDATEST was loaded and operated keeping the same conditions used for the tests made in CATLAB reactor, except for the use of $\mathrm{N}_{2}$ as both internal standard and balance gas instead of $\mathrm{He}$ and Ar. Further details on the OXIDATEST set-up and on the GC quantification method are provided by Sandoval-Bohorquez et al.[14].

\section{Results}

\subsection{Mass spectrometer calibration}

Figure 2 shows calibration curves for $\mathrm{CH}_{4}, \mathrm{CO}_{2}$, and $\mathrm{CO}$ for both the external (Figure 2a) and the internal (Figure 2b) standard methods. In all instances, the coefficient of determination $\left(\mathrm{R}^{2}\right)$ was higher than 0.995 . Therefore, Equations 11 and 12 are proved to be adequate for quantification with mass spectrometry. Overall, there was no obvious influence of the calibration method on the coefficient of determination. Thus, the flow of the gases entering the mass spectrometer was only affected by the setting of the automated flow controllers. Figure 2 also allows ruling out the overlapping of different $m / z$ signals. 

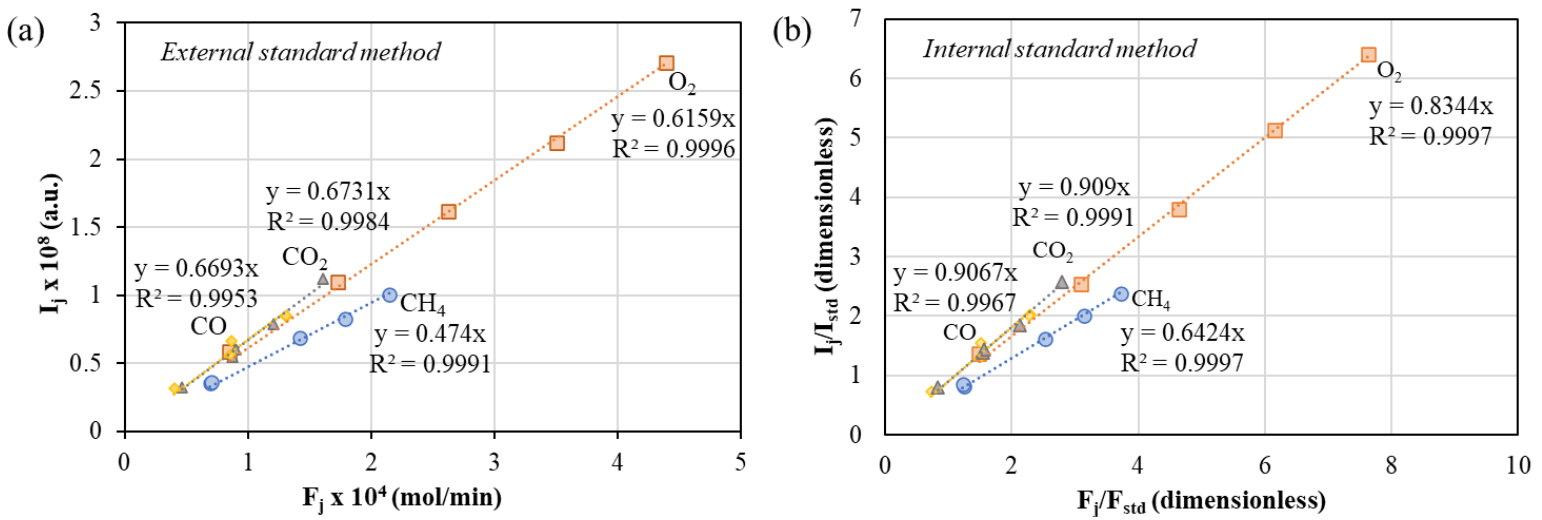

Figure 2. External (a) and internal (b) standard calibration of a gaseous mixture of $\mathrm{O}_{2}$ (orange), $\mathrm{CH}_{4}\left(\right.$ blue), $\mathrm{CO}_{2}$ (gray) and $\mathrm{CO}$ (yellow) by linear regression of mass spectrometer response signals, $\mathrm{I}_{\mathrm{j}}$, detected for different molar flow, Fj; for internal standard method, normalized response signals, $\mathrm{I}_{\mathrm{j}} / \mathrm{I}_{\mathrm{std}}$, and normalized molar flow, $\mathrm{F}_{\mathrm{j}} / \mathrm{F}_{\text {std }}$, were used. Argon was used as balance gas and helium was used as the internal standard. The inserts correspond to the equations of the straight trend line and the coefficient of determination from the regression analysis.

\subsection{On-line mass spectrometry characterization of the catalytic behavior}

Figure 3 shows the results of the catalytic combustion reaction quantification using external and internal standard methods, as follows: (a) response signals, (b) molar flows, and (c) conversions and selectivities, estimated by the external standard method, and (d) normalized response signals (e) normalized molar flows, and conversions and selectivities, estimated by the internal standard method. Figure 3a showed a trough between 100 and 360 min for the signals assigned to $\mathrm{O}_{2}$ and $\mathrm{CH}_{4}$ which is due to their consumption in the catalytic reaction. This trough coincided with a crest in the signals assigned to $\mathrm{CO}_{2}$ and $\mathrm{CO}$ due to their production in the catalytic reactor. All the signals assigned to the reaction gases decreased with time on stream. This is a typical observation for catalytic studies made with on-line mass spectrometry.[11] An analysis of Equations 1 and 2 suggests that this trend is due to a decrease of the pressure of the ion source due to the compensation made-up by the vacuum pump for allowing the gas stream entering the instrument. This trend was corrected after applying the normalization of the signals with the internal standard method, as shown in Figure 3d. Figures $3 \mathrm{~b}$ and e show the evolution of the molar flows from the reactor effluent, as estimated with the sensitivity factors presented earlier, using the external and internal standard methods, respectively. As it could be expected, the trends in these plots were the same as those followed by the response signals.

From the above results, the conversion of $\mathrm{CH}_{4}$ and $\mathrm{O}_{2}$, and the selectivities to $\mathrm{CO}_{2}$ and $\mathrm{CO}$ were calculated using both the external, Figure 3c, and the internal, Figure 3f, standard methods. For the external standard method some inconsistent trends were observed. First, an apparent steady increase in the conversions of both reactants after 200 min was observed. When interpreting the data, catalytic researchers may be led to think that this shows that the catalyst was "activated" during the test. Second, the selectivity to $\mathrm{CO}_{2}$ was larger than 1 at the beginning of the test. The inconsistencies found for the external standard method were not found with the internal standard method. 
Indeed, the conversions of $\mathrm{CH}_{4}$ and $\mathrm{O}_{2}$ remained stable after an initial period of deactivation of the catalyst, while the sum of the selectivities to $\mathrm{CO}_{2}$ and $\mathrm{CO}$ remained very close to 1 at all instances.
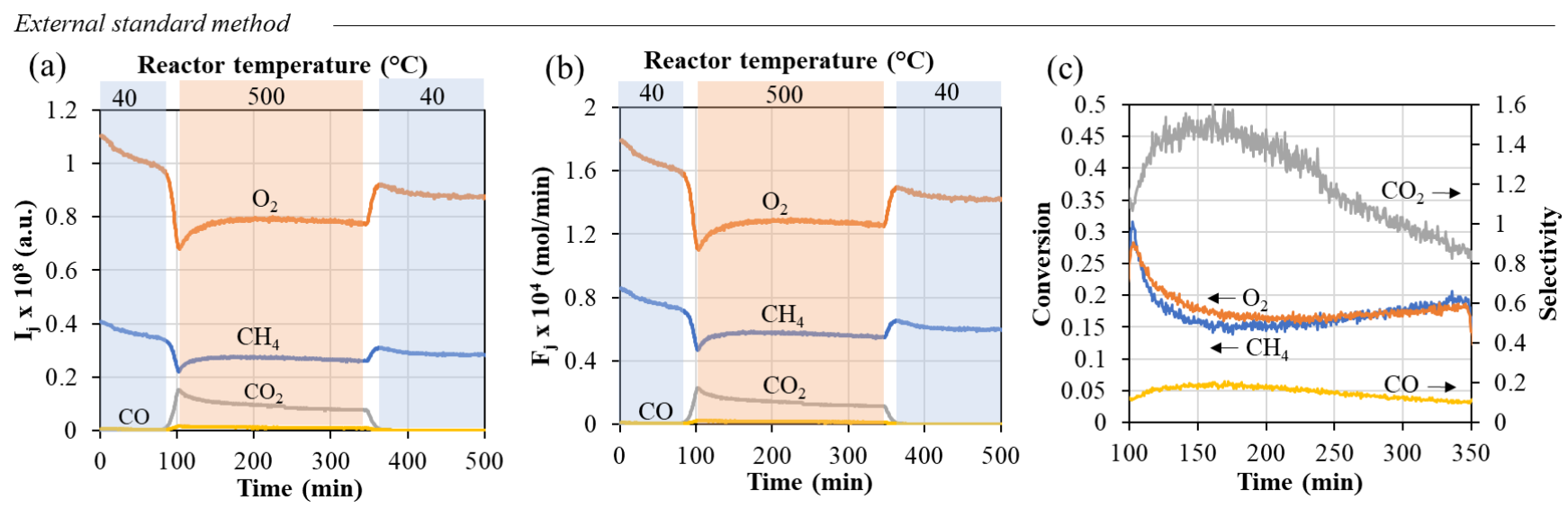

Internal standard method
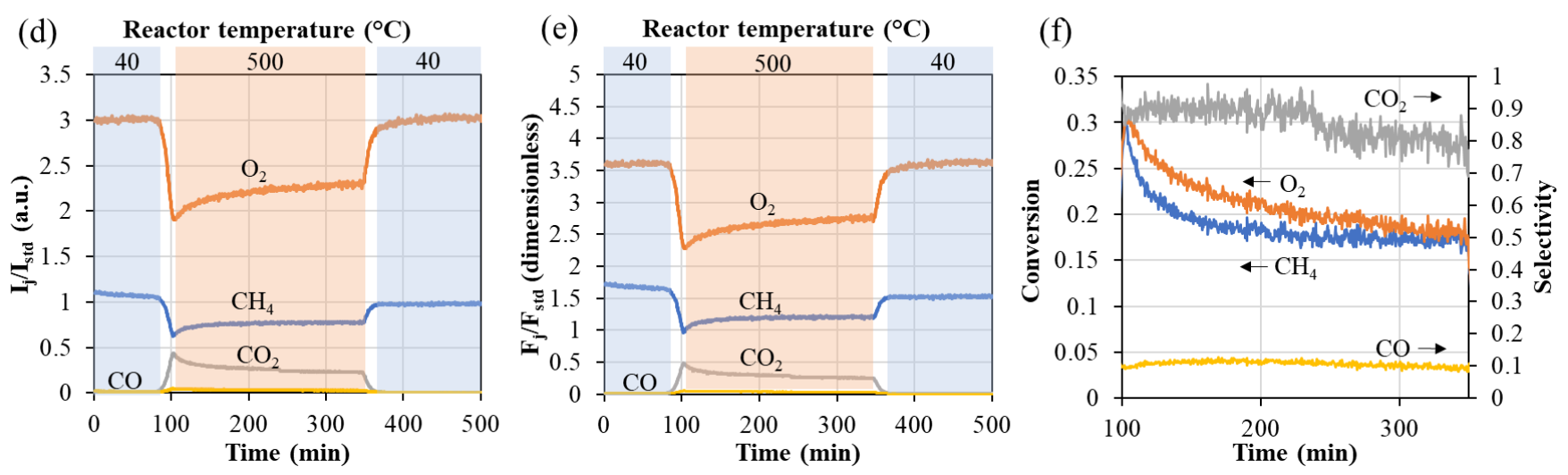

Figure 3. Comparison of the catalytic combustion reaction quantification using external and internal standard methods: (a) response signals, $\mathrm{I}_{\mathrm{j}}$, (b) molar flows, $\mathrm{F}_{\mathrm{j}}$, and (c) conversions and selectivities, estimated by the external standard method, and (d) normalized response signals, $\mathrm{I}_{\mathrm{j}} / \mathrm{I}_{\mathrm{std}}$, (e) normalized molar flows, $\mathrm{F}_{\mathrm{j}} / \mathrm{F}_{\text {std }}$, and conversions and selectivities, estimated by the internal standard method. Mass spectrometer response signals of $\mathrm{O}_{2}$ (orange), $\mathrm{CH}_{4}$ (blue), $\mathrm{CO}_{2}$ (gray), and $\mathrm{CO}$ (yellow) were continuously analyzed at the outlet of the catalytic reactor, which was programmed to run three stages: pre-reaction at $40{ }^{\circ} \mathrm{C}$, reaction at $500{ }^{\circ} \mathrm{C}$, and post-reaction at $40{ }^{\circ} \mathrm{C}$.

Figure 4 shows boxplots summarizing the estimated values of carbon mass balance coefficient after applying the external and internal standard methods. Accordingly, the external standard method produced values for the carbon mass balance coefficient whose average was $\sim 1.4$, and also showed the largest data dispersion. Conversely, the internal standard method had an average and median carbon mass balance coefficient of 1 with only slight variations throughout the experiments. Therefore, while the internal standard method appears highly reliable for the on-line characterization of catalytic experiments, the external standard method is highly unreliable for such a purpose. Furthermore, the conclusions drawn from the latter method are misleading for the interpretation of catalytic experiments. 


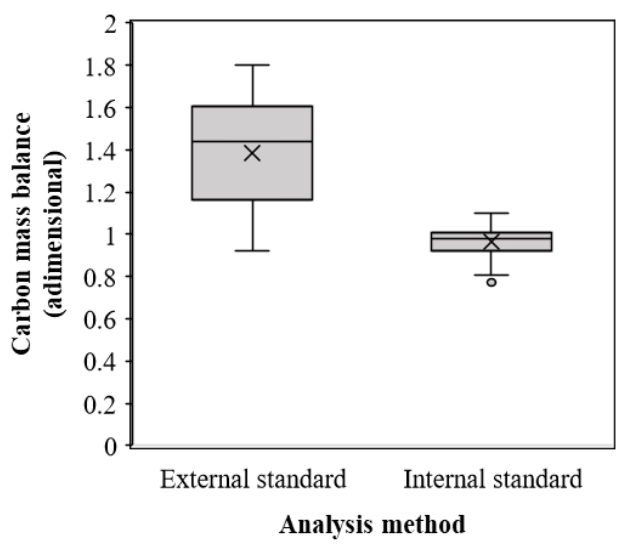

Figure 4. Boxplots comparing the carbon mass balance coefficients after applying the external and the internal standard methods for the on-line characterization of the continuous catalytic combustion of methane.

\subsection{Validation of the internal standard method}

The results from the on-line mass spectrometry quantification of the products from the catalytic combustion of methane were validated by comparing them with those obtained by on-line gas chromatography in the OXIDATEST set-up. Results from on-line gas chromatography in terms of FID and TCD areas of the reactants and products are presented in Figure 5. The similarity between both sets of results, conversions differed less than $3 \%$ between both types of measurements, validates the internal standard method for on-line mass spectrometry proposed in this work.
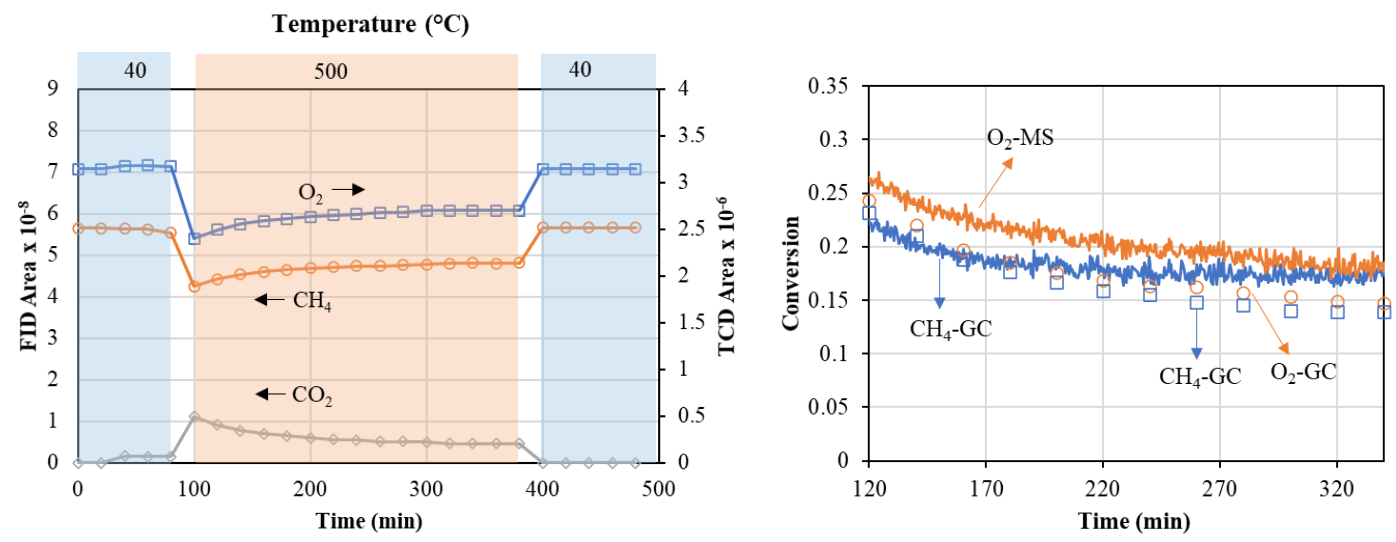

Figure 5. FID Areas of $\mathrm{CH}_{4}$ and $\mathrm{CO}_{2}$, and TCD Area of $\mathrm{O}_{2}$ detected by gas chromatography (GC) during the catalytic combustion of methane carried out in OXIDATEST (a), and comparison between conversions of $\mathrm{CH}_{4}\left(\right.$ blue) and $\mathrm{O}_{2}($ orange) obtained by GC and mass spectrometry (MS) (b).

\section{Conclusions}

Though on-line mass spectrometry is often used for the characterization of catalytic reactors, the quantification of the components of the products streams is still used in qualitative terms. Indeed, conventional data analysis in mass spectrometry to estimate key metrics such as conversion and selectivity is considered unreliable because of flow 
disturbances generated by changes in temperature or pressure in the coupled reactor-detector systems which in turn affect the intensity of the detected signal for the analyzed molecules. The results of this work demonstrated that such a behavior is present when quantification with mass spectrometry is made using the so-called external standard method of calibration. Conversely, the application of the internal standard method for quantification developed in this work produced a reliable characterization of the catalytic behavior for an experimental set-up consisting on an automated continuous fixed-bed flow reactor coupled on-line to a mass spectrometer detection system. For developing this method, mathematical expressions coupling the fundamentals of mass spectrometry detection and material balances were developed. The method was further validated with data obtained via the on-line gas chromatography characterization of a parent catalytic set-up. In general, the results of this work help provide a reliable and accurate basis for extending the use of on-line mass spectrometry in reaction process research.

\section{Acknowledgements}

This work was funded by Agencia Nacional de Hidrocarburos -ANH- and Minciencias, Colombia, within the frame of the projects 1102-721-50962: "Desarrollo de alternativas catalíticas para la reducción y valorización de emisiones de gases de efecto invernadero típicas de pozos y refinerías petroleras por combustión catalítica de VOCs y transformación de $\mathrm{CO}_{2}$ y CH4 en gas de síntesis" and 365-2018: "Preparación de nanomateriales basados

en metales de transición para procesos ligados al recobro térmico de crudos colombianos y análisis de su comportamiento catalítico en procesos de combustión in-situ."

\section{References}

[1] C. He, J. Cheng, X. Zhang, M. Douthwaite, S. Pattisson, Z. Hao, Recent Advances in the Catalytic Oxidation of Volatile Organic Compounds: A Review Based on Pollutant Sorts and Sources, Chem. Rev. 119 (2019) 4471-4568. https://doi.org/10.1021/acs.chemrev.8b00408.

[2] R. Rezaei, G. Moradi, S. Sharifnia, Dry Reforming of Methane over Ni-Cu/Al 2 O 3 Catalyst Coatings in a Microchannel Reactor: Modeling and Optimization Using Design of Experiments, Energy \& Fuels. 33 (2019) 6689-6706. https://doi.org/10.1021/acs.energyfuels.9b00692.

[3] H. Scott Fogler, Elements of chemical reaction engineering, Chem. Eng. Sci. 42 (1987) 2493. https://doi.org/10.1016/0009-2509(87)80130-6.

[4] J. Haber, Manual on catalyst characterization (Recommendations 1991), Pure Appl. Chem. 63 (1991) 1227-1246. https://doi.org/10.1351/pac199163091227.

[5] L. He, Y. Fan, J. Bellettre, J. Yue, L. Luo, A review on catalytic methane combustion at low temperatures: Catalysts, mechanisms, reaction conditions and reactor designs, Renew. Sustain. Energy Rev. 119 (2020). https://doi.org/10.1016/j.rser.2019.109589. 
[6] R. Andersson, M. Boutonnet, S. Järås, On-line gas chromatographic analysis of higher alcohol synthesis products from syngas, J. Chromatogr. A. 1247 (2012) 134-145. https://doi.org/10.1016/j.chroma.2012.05.060.

[7] A. Ray, T. Bristow, C. Whitmore, J. Mosely, On-line reaction monitoring by mass spectrometry, modern approaches for the analysis of chemical reactions, Mass Spectrom. Rev. 37 (2018) 565-579. https://doi.org/10.1002/mas.21539.

[8] J.H. Batey, The physics and technology of quadrupole mass spectrometers, Vacuum. 101 (2014) 410-415. https://doi.org/10.1016/j.vacuum.2013.05.005.

[9] J.A. Olivares, Mass Spectrometry in Process Analysis, in: Encycl. Anal. Chem., John Wiley \& Sons, Ltd, Chichester, UK, 2006: pp. 1-16. https://doi.org/10.1002/9780470027318.a2109.

[10] R.I. Kaiser, P. Jansen, K. Petersen, K. Roessler, On line and in situ quantification of gas mixtures by matrix interval algebra assisted quadrupole mass spectrometry, Rev. Sci. Instrum. 66 (1995) 5226-5231. https://doi.org/10.1063/1.1146089.

[11] Z. Cheng, T. Mozammel, J. Patel, W.J. Lee, S. Huang, S. Lim, X. Ma, S. Bhargava, C. Li, A method for the quantitative analysis of gaseous mixtures by online mass spectrometry, Int. J. Mass Spectrom. 434 (2018) 23-28. https://doi.org/10.1016/j.ijms.2018.09.002.

[12] R. Whelpton, Quality assurance | internal standards, 3rd ed., Elsevier Inc., 2019. https://doi.org/10.1016/B978-0-12-409547-2.14399-9.

[13] C. Wang, E. Rosenfeldt, Y. Li, R. Hofmann, External standard calibration method to measure the hydroxyl radical scavenging capacity of water samples, Environ. Sci. Technol. 54 (2020) 1929-1937. https://doi.org/10.1021/acs.est.9b06273.

[14] V.S. Sandoval-Bohorquez, E.A.V. Rozo, V.G. Baldovino-Medrano, A method for the highly accurate quantification of gas streams by on-line chromatography, J. Chromatogr. A. 1626 (2020) 461355. https://doi.org/10.1016/j.chroma.2020.461355.

[15] N. Holmes, G.R. Akien, R.J.D. Savage, C. Stanetty, I.R. Baxendale, A.J. Blacker, B.A. Taylor, R.L. Woodward, R.E. Meadows, R.A. Bourne, Online quantitative mass spectrometry for the rapid adaptive optimisation of automated flow reactors, React. Chem. Eng. 1 (2016) 96-100. https://doi.org/10.1039/c5re00083a.

[16] L.L. Hohrenk, F. Itzel, N. Baetz, J. Tuerk, M. Vosough, T.C. Schmidt, Comparison of Software Tools for Liquid Chromatography-High-Resolution Mass Spectrometry Data Processing in Nontarget Screening of 
Environmental Samples, Anal. Chem. 92 (2020) 1898-1907.

https://doi.org/10.1021/acs.analchem.9b04095.

[17] R. Helmus, T.L. ter Laak, A.P. van Wezel, P. de Voogt, E.L. Schymanski, patRoon: open source software platform for environmental mass spectrometry based non-target screening, J. Cheminform. 13 (2021) 125. https://doi.org/10.1186/s13321-020-00477-w.

[18] G. Analysis, T. Analysis, R.G. Analysis, Software for Hiden Mass Spectrometers, (n.d.). https://www.hidenanalytical.com/wp-content/uploads/2020/06/MASsoft-PC-Win-10-Software.pdf.

[19] J.T. Watson, O.D. Sparkman, Introduction to Mass Spectrometry, Fourth Edi, John Wiley \& Sons, Ltd, Chichester, UK, 2007. https://doi.org/10.1002/9780470516898.

[20] E. De Hoffmann, V. Stroobant, Mass Spectrometry Principles and Applications Third Edition, 2007. https://www.wiley.com/en-us/Mass+Spectrometry\%3A+Principles+and+Applications\%2C+3rd+Editionp-9780470033111.

[21] R.E. Ellefson, D. Cain, C.N. Lindsay, Calibration of mass spectrometers for quantitative gas mixture analysis, J. Vac. Sci. Technol. A Vacuum, Surfaces, Film. 5 (1987) 134-139. https://doi.org/10.1116/1.574126.

[22] J.P. Hammond, Quality Assurance: Spectroscopic Standards, in: Ref. Modul. Chem. Mol. Sci. Chem. Eng., Elsevier, 2018: pp. 426-432. https://doi.org/10.1016/B978-0-12-409547-2.00455-8.

[23] K.D. Cook, K.H. Bennett, M.L. Haddix, On-line mass spectrometry: A faster route to process monitoring and control, Ind. Eng. Chem. Res. 38 (1999) 1192-1204. https://doi.org/10.1021/ie9707984.

[24] E. Heinzle, A. Oeggerli, B. Dettwiler, On-line fermentation gas analysis: Error analysis and application of mass spectrometry, Anal. Chim. Acta. 238 (1990) 101-115. https://doi.org/10.1016/S00032670(00)80528-0.

[25] B.S. Ferreira, F. Van Keulen, M.M.R. Da Fonseca, Novel calibration method for mass spectrometers for on-line gas analysis. Set-up for the monitoring of a bacterial fermentation, Bioprocess Eng. 19 (1998) 289296. https://doi.org/10.1007/s004490050522.

[26] E. Heinzle, J. Moes, M. Griot, H. Kramer, I.J. Dunn, J.R. Bourne, On-line mass spectrometry in fermentation, Anal. Chim. Acta. 163 (1984) 219-229. https://doi.org/10.1016/S0003-2670(00)81510-X.

[27] C.N. Satterfield, Acid and Zeolite Catalysts, Heterog. Catal. Ind. Pract. (1991) 209-266. 